\title{
Computational study of cavity flowfield at transonic speeds
}

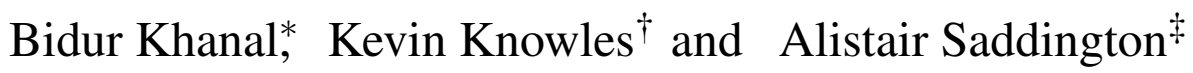 \\ Cranfield University \\ Defence Academy of the United Kingdom \\ Shrivenham, SN6 8LA, England
}

In this paper, the results of a computational study on the unsteady flow features in three-dimensional empty cavities and a cavity with a store are presented. Flow simulations with a turbulence model based on a hybrid method, which behaves as a standard RANS model within the attached boundary layer and as a LES Sub-Grid Scale model in the rest of the flow, including the separated regions, are used in this study. The time-mean flow study showed the existence of spanwise flow in the 3D cavity. In the unsteady flow study, computed near-field acoustic spectra were for empty cavity as well as cavity-withstore cases. Unsteady results from an empty cavity case are compared with experimental data and the frequency of the dominant mode is in good agreement with the experiment. Study of unsteady pressure spectra for the cavity-with-store case found the presence of many peaks and the corresponding mode frequencies were found to agree well with the Rossiter modes. The mean flowfield visualisation for the cavity-with-store case clearly showed that the mean flowfield was effectively divided into two halves with signifiant reduction of the spanwise flow across the cavity width. This blockage effect of store and strut on the spanwise flow is thought to have reduced the interaction, and subsequent non-linear coupling between, the Rossiter modes. This may be the reason for the coexistence of multiple modes without the coupling among them.

\footnotetext{
${ }^{*}$ Research Student; Aeromechanical Systems Group; Student member AIAA

${ }^{\dagger}$ Professor and Head of Aeromechanical Systems; Associate Fellow AIAA

†Lecturer; Aeromechanical Systems Group
} 


\section{Introduction}

Cavities or surface cutouts exist commonly in aerospace applications and are also well documented in aerodynamic noise study. Flow over a simple cavity geometry produces complex features consisting of flow induced resonant tones, multiple flow and acoustic instabilities, and wave interactions. Cavity flowfields are widely present in many aerospace applications, such as wheel wells, weapons bays, and other fuselage openings. Internal weapons bays on military aircraft, in particular, have several design advantages. Reduced aerodynamic drag, low radar cross-section and avoidance of aerodynamic heating of the store are a few examples of these. However, once the weapons bay doors are opened, the resultant flowfield is dependent on the cavity geometry and the freestream flow conditions. Unsteady flow approaching the cavity forms an oscillating free shear layer when the flow separates from the upstream edge of the cavity. The resultant flowfield then interacts with the stores at the beginning of the store release leading to several undesirable aerodynamic effects. Firstly, the self-sustaining pressure fluctuations in the cavity cause cavity resonance which in turn leads to structural fatigue of the aircraft and the store. Secondly, the interaction of the store with the unsteady shear layer formed over the cavity can lead to unpredicted motion of the store. Hence, study of cavity flowfield is necessary to understand these phenomena.

Numerous experimental and computational research studies ${ }^{1-5}$ have been carried out to understand complex flow features existing in cavities and many more are continuing to understand the cause behind such complex flowfields and suppressing the resonant tones. Several computational studies in the past have experienced a limitation with Reynolds-averaged Navier-Stokes (RANS) and turbulence models applied to unsteady flows. ${ }^{6-8}$ The RANS solver produces increased eddy viscosity which causes excessive damping of the unsteadiness of the flowfield. This is due to the assumption in the RANS turbulence model that all scales of the unsteady motion are to be captured and modelled by it. Spatially filtered models such as Large-Eddy Simulation (LES) have provided improved results for simulating unsteady flows. LES models, however, are currently limited to low Reynolds numbers because of the computing resources required to resolve the small-scale turbulent structures. LES is, therefore, not a feasible tool yet for the study of cavity flowfields at transonic 
speeds. Recently, hybrid methods which behave as a standard RANS model within the attached boundary layer and as a LES Sub-Grid Scale model in the rest of the flow, including the separated regions, have been introduced to address this problem., 6,9 Flow simulations with a turbulence model based on such a hybrid method are used in this study.

Cavity flows are usually classified by the length-to-depth ratio $(L / D)$ of the cavity and four different types of cavity flows are categorized depending on the value of this ratio. Early studies based on the analysis of wake flows by Charwat et al. ${ }^{10,11}$ led to the characterisation of three distinct types of cavity flows based on the cavity floor pressure distributions which are primarily dependent on the geometry of the cavity. These were categorized as open, closed and intermediate flow. In the 1980s, Stallings and Wilcox ${ }^{4}$ studied the cavity pressure distribution at supersonic speeds experimentally. Their work led to a more detailed classification of the flow types and the intermediate flow type was further explained and divided into transitional open and transitional closed flow. Cavity flows exhibit a wide variety of phenomena whose precise nature depends sensitively on a number of parameters including the value of $L / D$. In the literature, vastly different values of $L / D$ have been quoted to define the flow types. For instance: $L / D<7$ for open and $L / D>13$ for closed cavity flow is reported in ${ }^{4}$ whereas $L / D<10$ for open and $L / D>13$ for closed cavity flow is reported in. ${ }^{12}$ Similarly, Dix and Bauer ${ }^{1}$ quote $L / D<9$ for open and $L / D>$ 13 for closed cavity flow whereas Srinivisan and Baysal ${ }^{2}$ quote $L / D<3$ for open and $L / D>10$ for closed cavity flowfields. Tracy and Plentovich ${ }^{13}$ investigated the variations in the values of $L / D$ and concluded that the vast disagreements in the literature were due to the dependence of the cavity flow type on Mach number as well as $L / D$. The other important of parameters that affect cavity flow types are incoming boundary layer thickness $(\delta)$, ambient density $(\rho)$, viscosity $(\mu)$, and speed of sound $\left(a_{0}\right)$. Although the underlying physical mechanisms vary, it is known that selfsustaining oscillations develop over a wide range of these parameters. In this paper results from the computational investigations of open cavity flows are presented. 


\section{Computational Setup}

Two types of 3D geometries were considered in this investigation. The first type is a threedimensional plane cavity, which comprises a simple rectangular cutout in an otherwise infinite plate and is therefore fully described by its length-to-depth and length-to-width ratios. Hereafter the plane cavity geometry will also be referred as the clean cavity. The second type had a store in the cavity as shown in Figure 1. The first geometry considered was a $16 \mathrm{~cm}$ long clean cavity case with an $L / D$ of 5 and $W / D$ of 2.5 and it had identical flow and geometrical settings to the experimental study of Ritchie. ${ }^{14}$ The second case was a $12.2 \mathrm{~cm}$ deep cavity with an $L / D$ of 5.42 and $W / D$ of 2.0 and the third case had same cavity dimensions as the second but it also had a store in the cavity. The last two cavity geometries correspond to some of the many cases in the experimental investigation by Tracy and Plentovich. ${ }^{13,15-17}$ Again, as with the geometry, the flow condition corresponds to a subset of those investigated by Tracy and Plentovich.

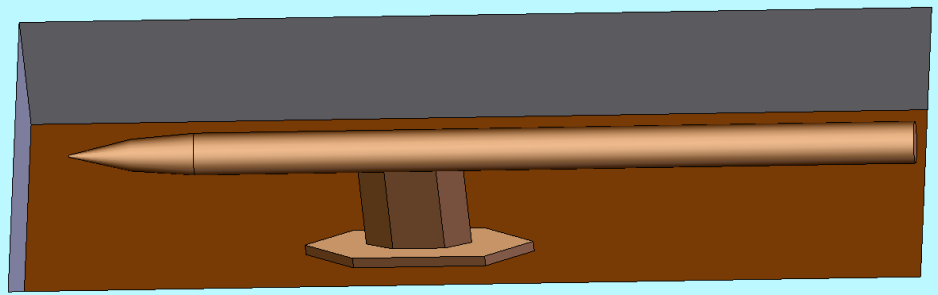

Figure 1. Isometric view of the 3D cavity with store.

The three-dimensional computational domain extends $4 L$ upstream, $5 L$ downstream and $3 L$ vertically and laterally from the cavity. The overall mesh size for all the cases was maintained approximately between 2.0 million cells to 2.75 million cells. Grid cells were clustered along all solid walls and in the region of the shear layer using a hyperbolic-tangent distribution. At the cavity leading edge, the boundary layer is resolved with approximately 20 mesh points. Figure 2 
shows the section views of the grids in Cavity-with-store and empty cavity cases.

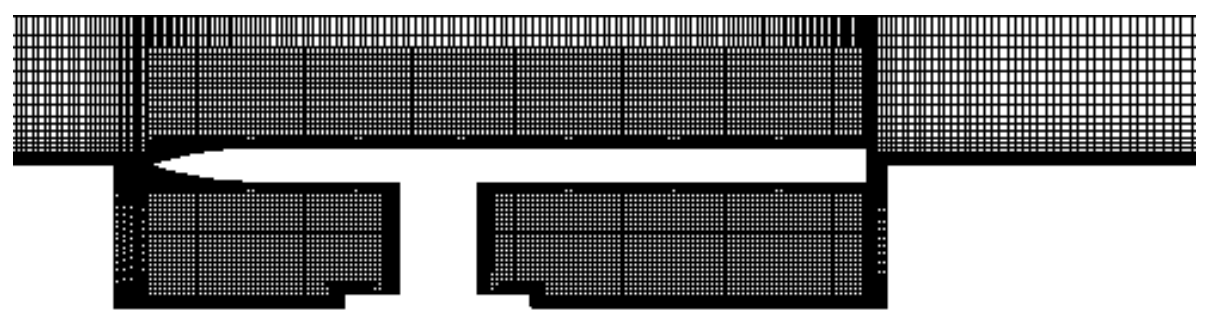

(a) Section view of Cavity-with-store grid.

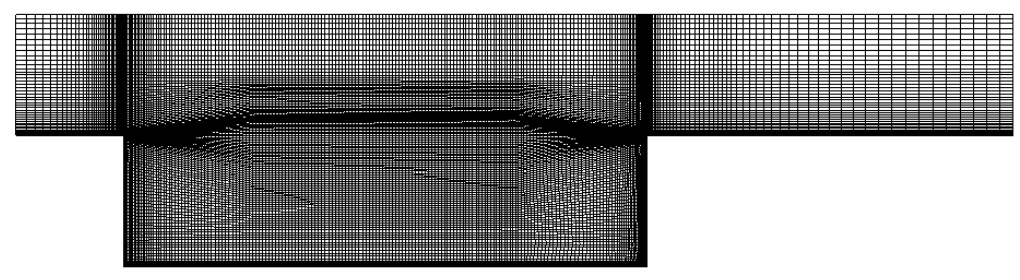

(b) grid distribution in empty cavity.

Figure 2. Structured grid distribution.

The CFD solver FLUENT was used to simulate all the cavity cases considered in this paper. FLUENT is a finite-volume solver and the temporal and spatial discretization schemes available in it provide at most second-order accuracy in space and time. Many studies ${ }^{18,19}$ have shown FLUENT to be capable of simulating unsteady flow problems, and of resolving the flow structures responsible for noise generation when suitably designed computational mesh and time-step sizes are used. Options for both explicit as well as the implicit time-stepping are available with the solver. 


\section{Results}

\section{A. Computed Results}

\section{Cavity time-mean flow}

A study of the time-mean flowfield shows that the time-mean flow is not uniform across the cavity span. This can be concluded from Figure 3 which shows the variation of mean $C_{p}$ for the first cavity case along with experimental $C_{p}$ data at floor centreline. For this case, the centerline of the cavity does not record the greatest variation in the pressure coefficient. Instead, the greatest variation is found at a position $16.7 \%$ from the centreline. This is thought to be the result of the significant spanwise flow present in wide $3 \mathrm{D}$ cavities. Computed $C_{p}$ showed some discrepancies with the experiment in the rear half of the cavity. The Figure 4 shows the time-mean streamlines for this case. The streamlines can be seen to cause stronger downflow on either side of the cavity centreline.

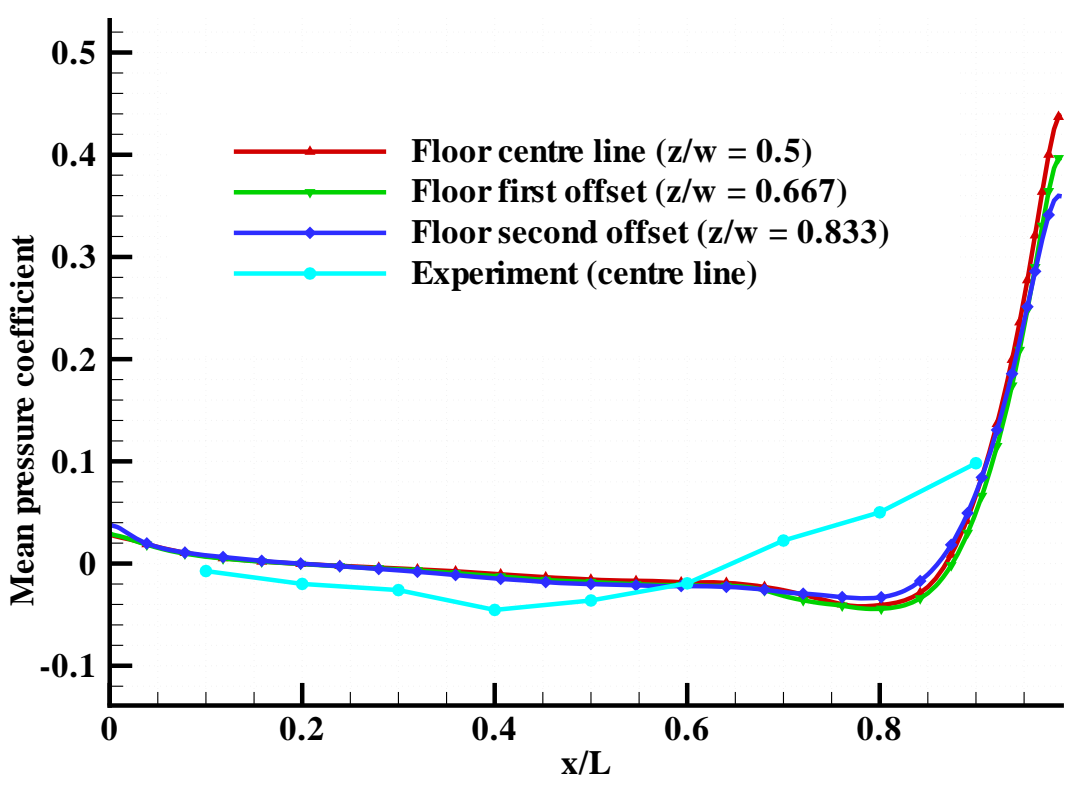

Figure 3. Mean $C_{p}$ along varying spanwise locations, $L / D=5$ and $W / D=2.5$.

Mean $C_{p}$ plots are also compared for the cavity with an $L / D$ ratio of 5.42 . This case was simulated in clean configuration as well as with a store in the cavity. The flow conditions were set to match the experimental study of Stallings et al. ${ }^{15}$ CFD results and experimental data are 


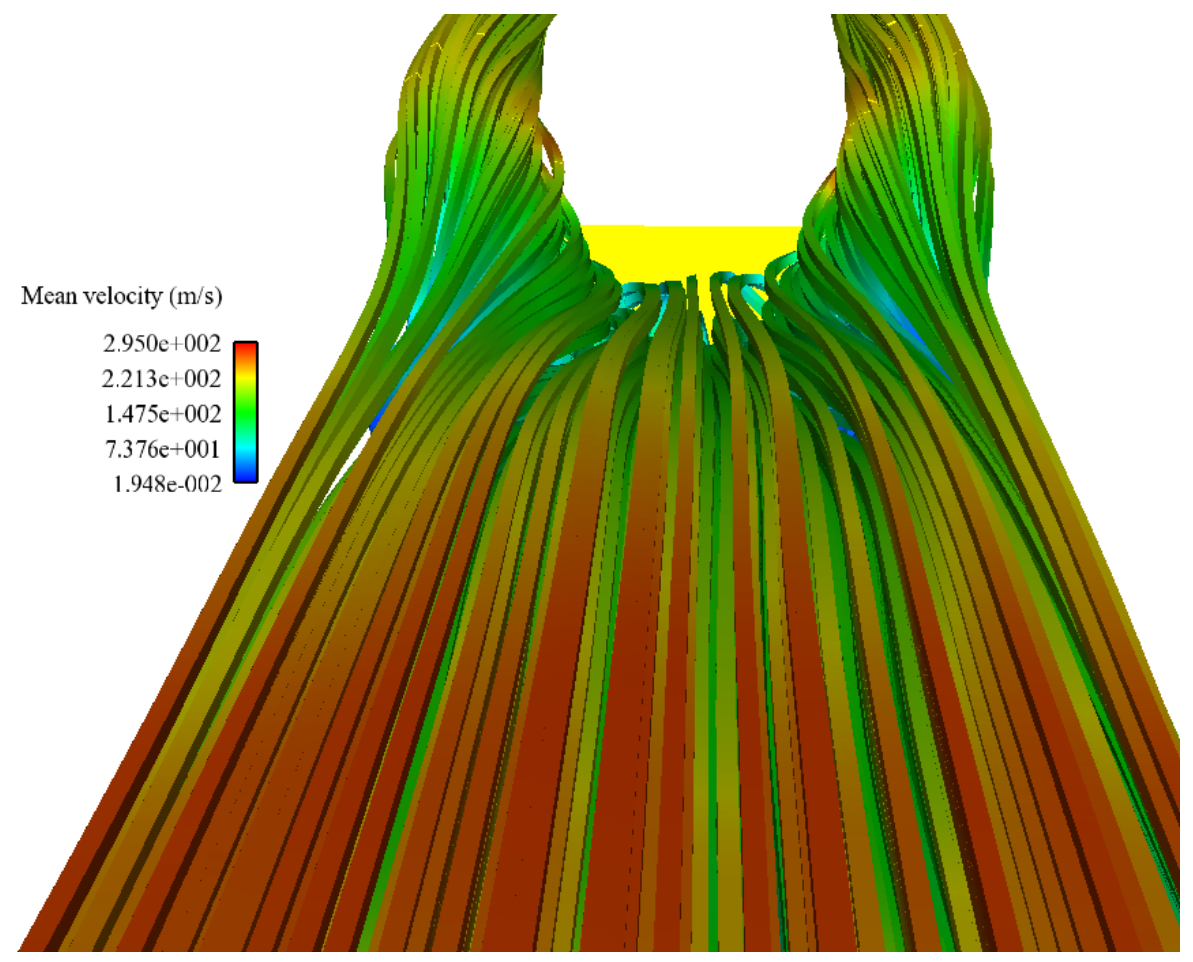

(a) View from the top.

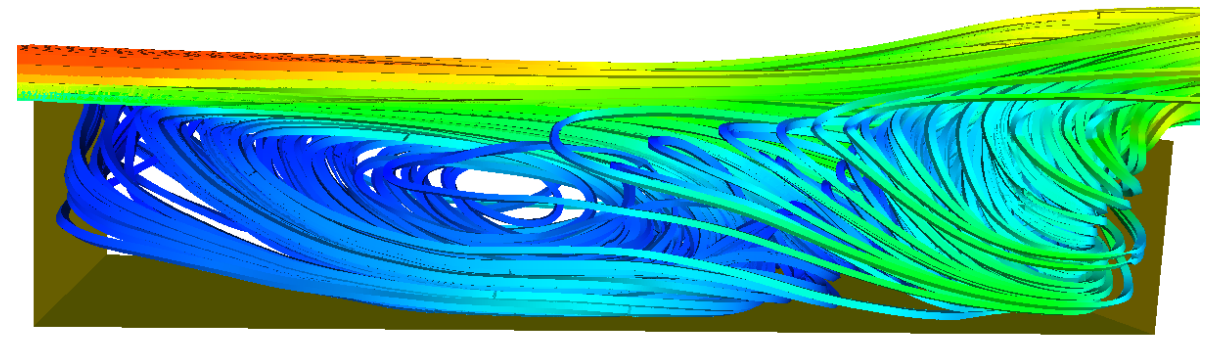

(b) View from the side.

Figure 4. Time-mean streamlines for 3D cavity, $L / D=5$ and $W / D=2.5$. 
compared in Figure 5. From the figure, it can be seen that there is negligible effect on mean $C_{p}$ due to the presence of the store in the front half along the cavity length. Computed results over-predicted the experiment by no more than 0.025 in the front half. The rear half of the cavity showed small effect on $C_{p}$ plot due to the presence of store. The computed result slightly overpredicted the experiment but the curves have similar characteristics (i.e. similar slope). Overall the computed mean flowfield agreed reasonably well with the experiment. Mean streamlines were also visualised for the cavity-with-store case to understand the time development of the mean flow due to the presence of store. Figure 6 shows the streamlines for the store case. The streamlines are seen to have divided due to the presence of the store and the supporting strut. The view from the rear of the cavity clearly shows the streamlines rolling up in two halves on the either side of the store body.

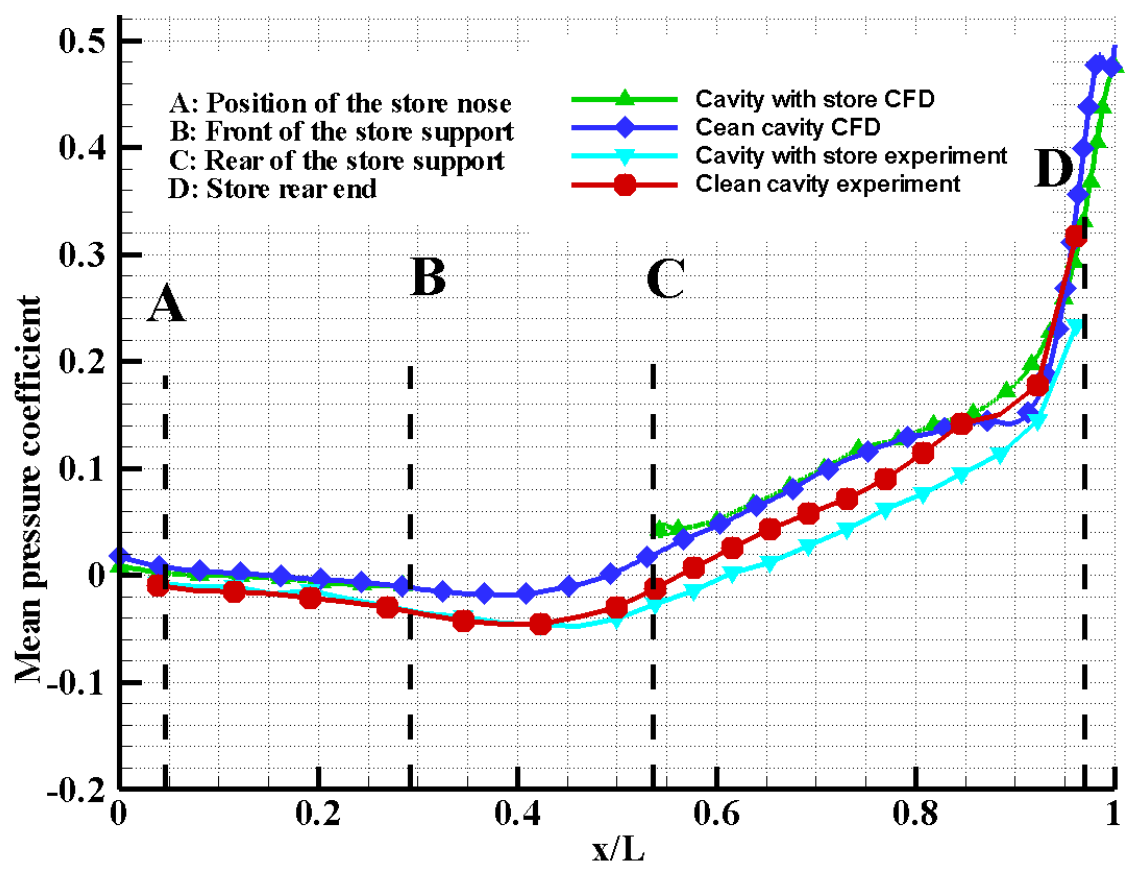

Figure 5. Mean $C_{p}$ variations for clean cavity and cavity-with-store cases.

\section{Time-dependent flowfield}

Unsteady computations were performed for all three cavity cases. Figure 7 shows the instantaneous iso-surfaces of vorticity magnitude and the second invariant of the velocities ${ }^{20}$ for the first case. 


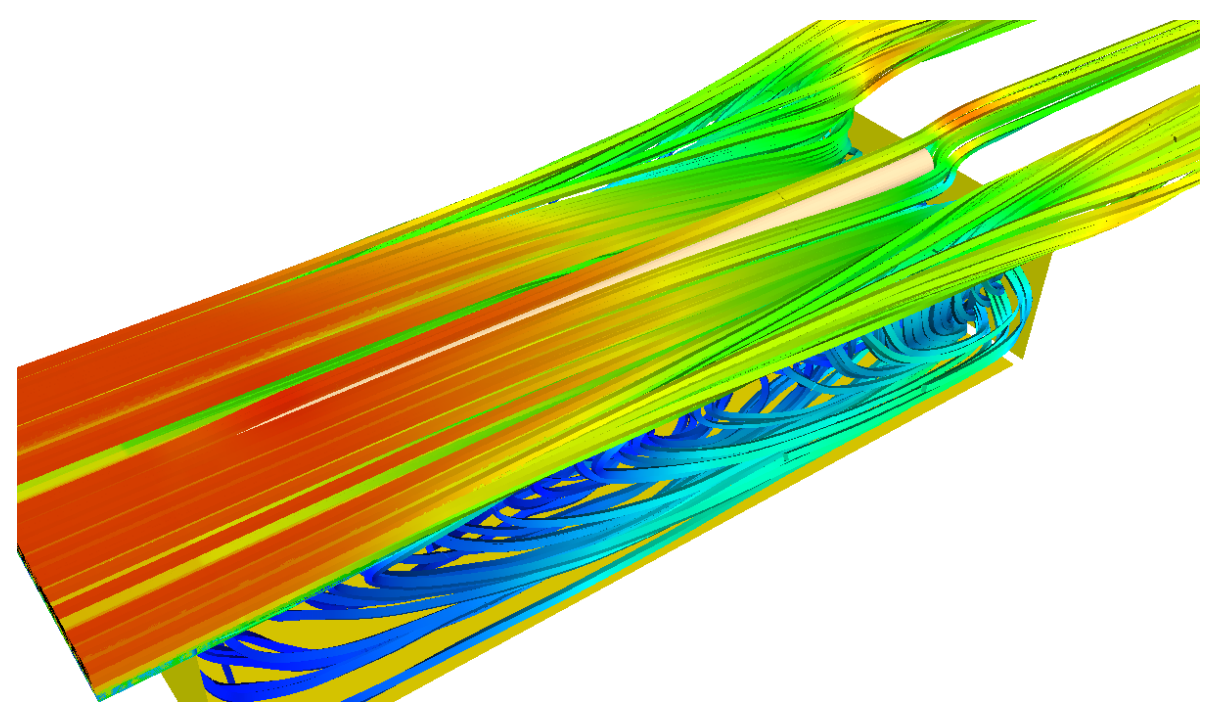

(a) View from the top.

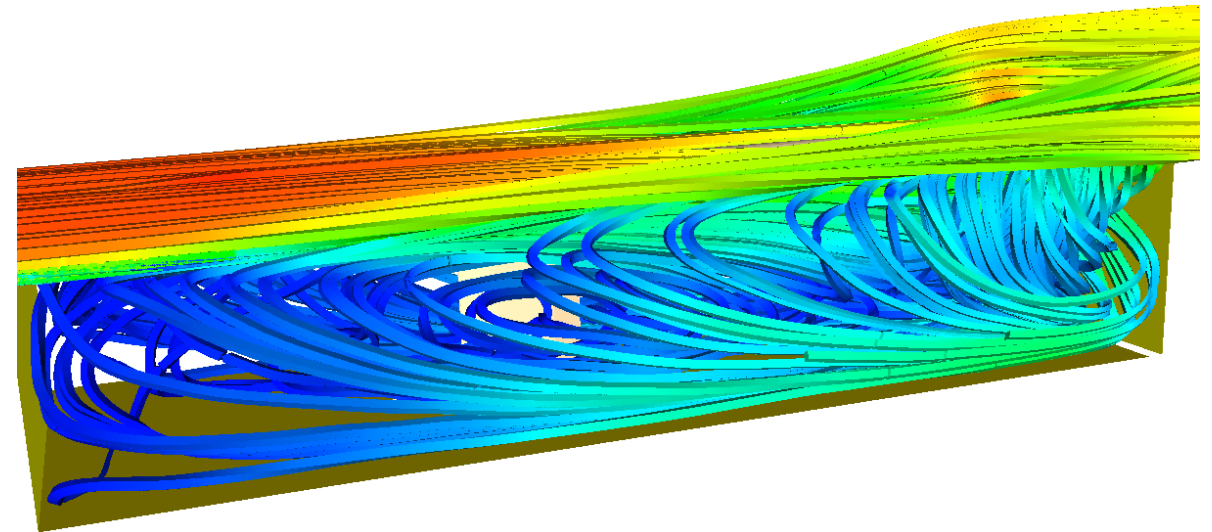

(b) View from the side.

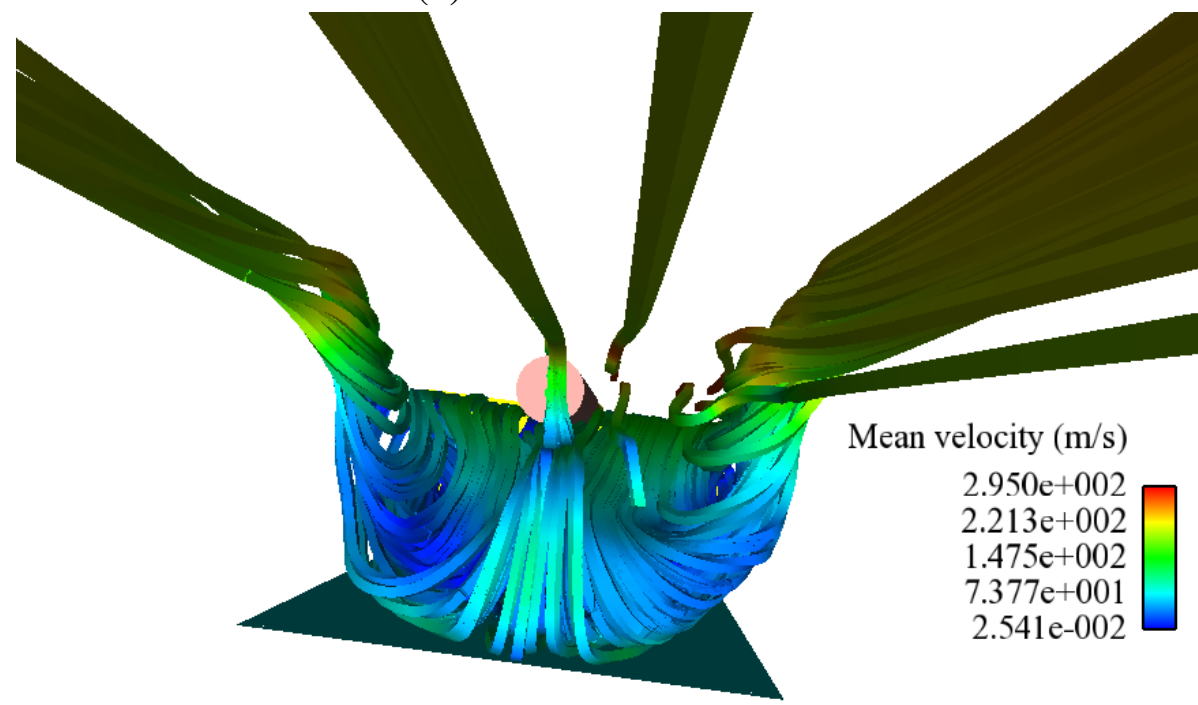

(c) View from the cavity rear wall.

Figure 6. Time-mean streamlines for 3D cavity-with -store, $L / D=5.42$ and $W / D=2$.

\section{9 of 18}

Computational study of cavity flowfield at transonic speeds, Khanal et al. 
The presence of large scale structures can clearly be seen from the figure.

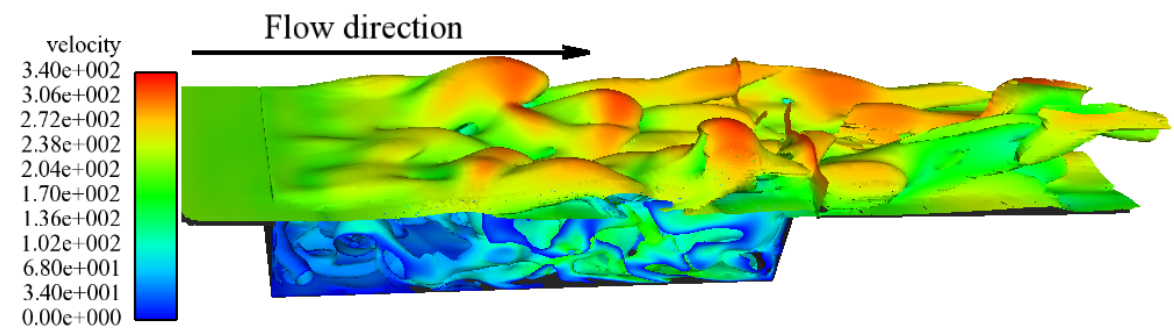

(a) Vorticity magnitude.

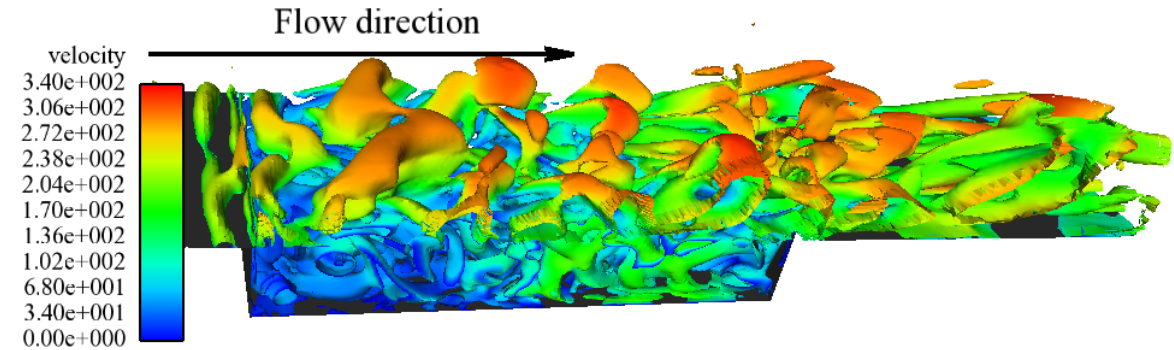

(b) Second invariant of the velocities.

Figure 7. Iso-surfaces showing flow structure in the cavity.

To study the unsteady flow quantitatively, the frequency content of the nearfield pressure fluctuations were extracted using fast fourier transforms. Figure 8 shows the comparison between experimental and computed pressure spectra. From the comparison, the frequency of the dominant mode is well predicted by the computation compared to experiment.

The last two cavity cases studied had a $L / D$ ratio of 5.42 and $W / D$ ratio of 2 . One of the two was an empty cavity whereas the other had a store with a supporting strut. The primary effect of the $3 \mathrm{D}$ geometry is seen to introduce a warping of the vortex axis across the span of the cavity. This seems to be the result of the end walls retarding the growth of the vortical structures as they move downstream of the cavity. Figures 9 and 10 show the iso-surfaces of the vorticity magnitude for the clean cavity and the cavity with the store respectively. From the figures, the warping of the vorticity iso-surface can be seen to be enhanced due to presence of the store. As the vortical structures in these regions reach the trailing edge, the vortex axis is aligned with the streamwise direction. Then the $3 \mathrm{D}$ flow in the end wall regions leads to the formation of thin streamwise vortices which is also clear from the study of time-mean streamlines in Figures 4 and 6. 


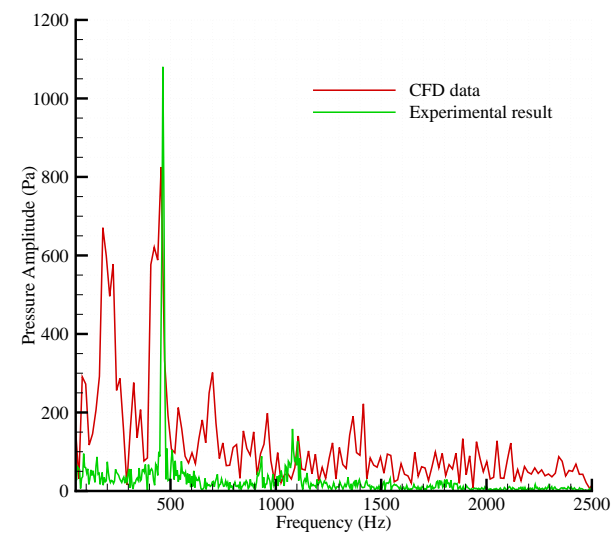

(a) Floor at $\mathrm{x} / \mathrm{L}=0.10$.

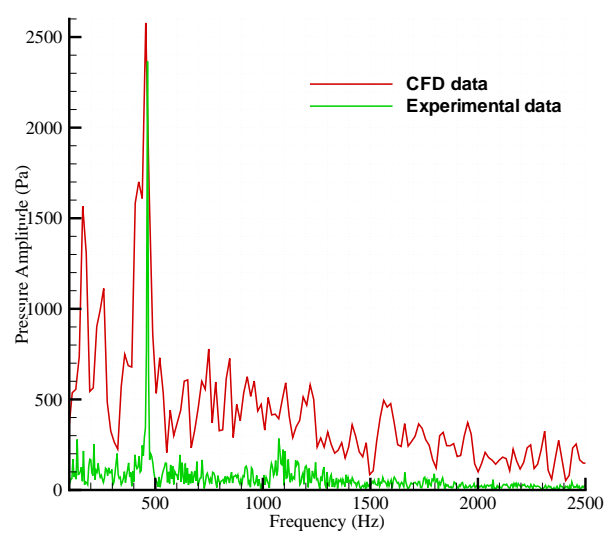

(b) Floor at $\mathrm{x} / \mathrm{L}=0.90$.

Figure 8. Empty cavity pressure spectra at floor centreline, $L / D=5$ and $W / D=2.5$.

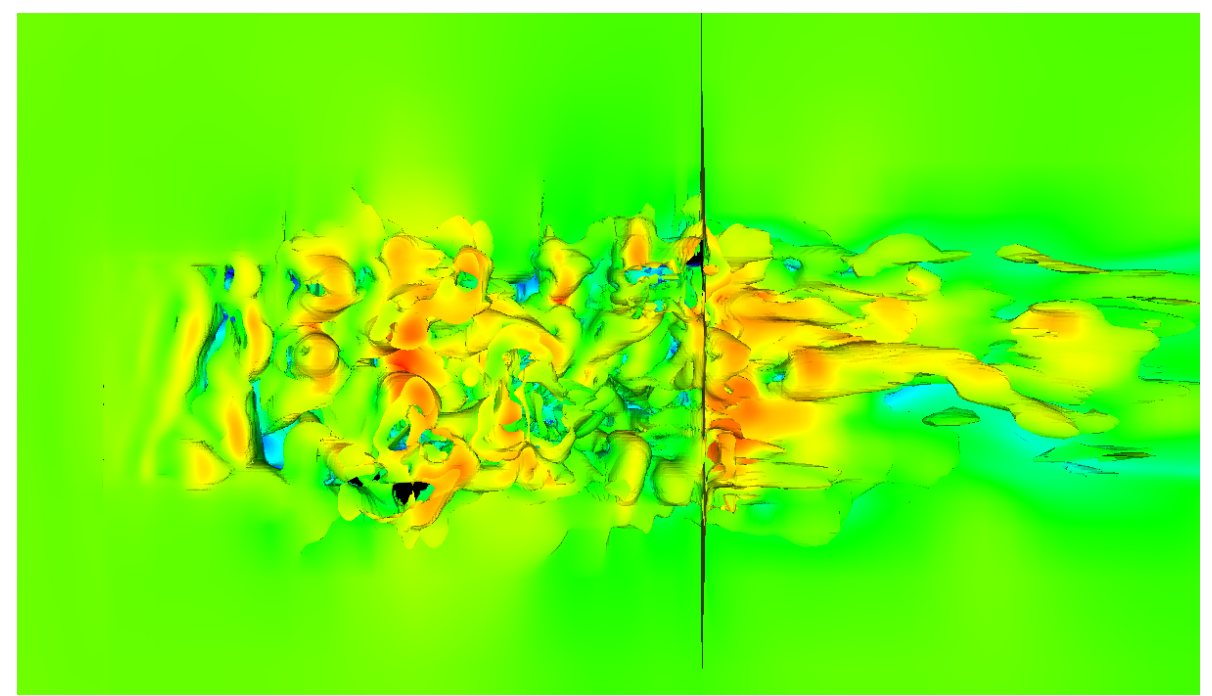

Figure 9. Vorticity iso-surface, view from the top of 3D clean cavity, $L / D=5.42$ and $W / D=2$. 


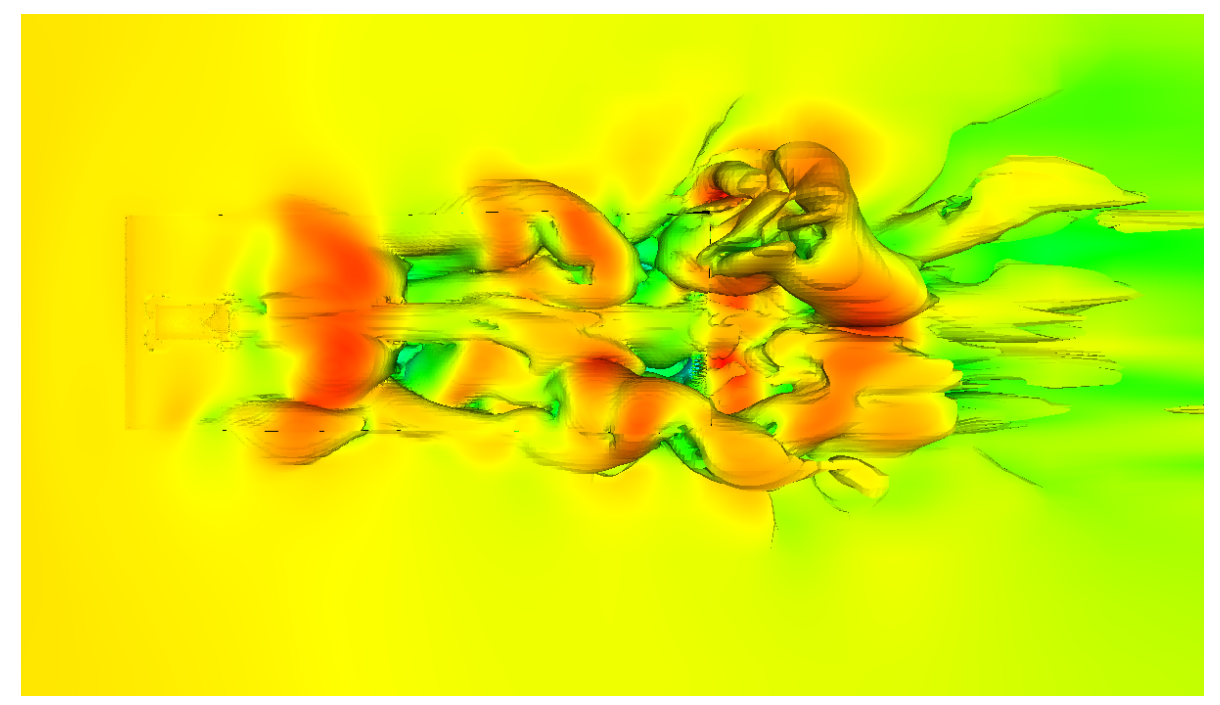

Figure 10. Vorticity iso-surface, view from the top of 3D cavity with a store, $L / D=5.42$ and $W / D=2$.

Figures 11, 12 and 13 show the frequency content of the pressure signals for clean cavity and the cavity-with-store. Figure 14 compares the pressure spectra from the two cases along the cavity centreline.

Predicted mode frequencies from the two cases along with the calculation using the modified Rossiter formula is presented in Table 1. From the results, the first mode frequency from the clean cavity case is found to agree well with modified Rossiter formula. The dominant mode, however, is not the first mode and the dominant peak occurs at $212 \mathrm{~Hz}$. This frequency is significantly lower compared to the second mode of $279 \mathrm{~Hz}$ predicted by the modified Rossiter formula. Higher order modes are absent in the spectra. The cavity-with-store case, however, has many peaks in the pressure spectra and the corresponding mode frequencies agree well with the Rossiter formula. Up to the five Rossiter modes are clearly present for the store case in contrast to the clean cavity which only had first and second modes present. High speed cavity flows are characterised by the existence of more than single dominant modes and these multi-modes are found to couple nonlinearly resulting in amplitude modulations at different frequencies. This is most probable for the clean cavity because spanwise flow fluctuations (i.e in the z-axis direction) are dominant compared to the store case which has less pronounced spanwise flow. Hence a coupling between the lateral and longitudinal instabilities may have resulted in a dominant peak at $212 \mathrm{~Hz}$ for the clean cavity 


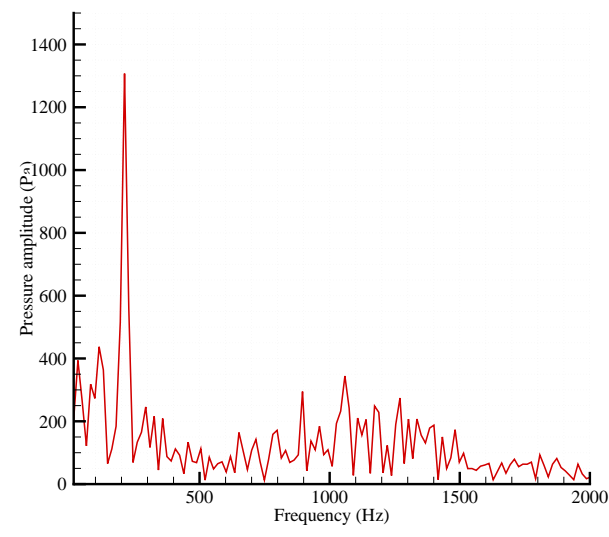

(a) Floor at $\mathrm{x} / \mathrm{L}=0.10$.

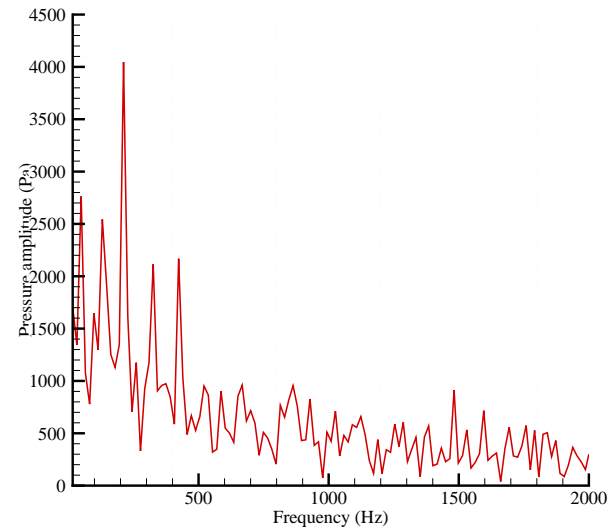

(c) Rear cavity wall at $\mathrm{y} / \mathrm{D}=0.25$.

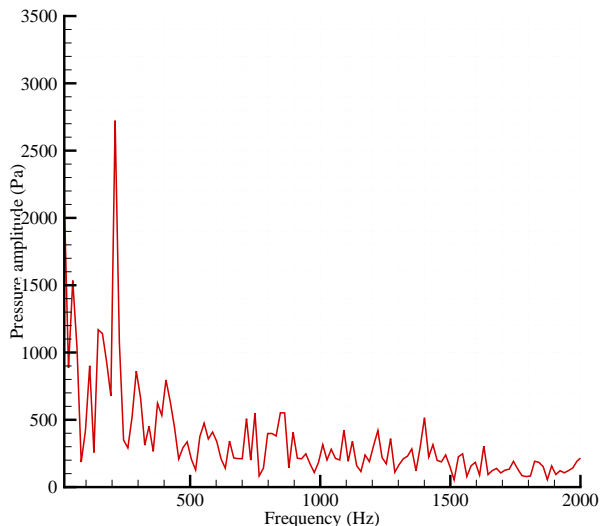

(b) Floor at $\mathrm{x} / \mathrm{L}=0.90$.

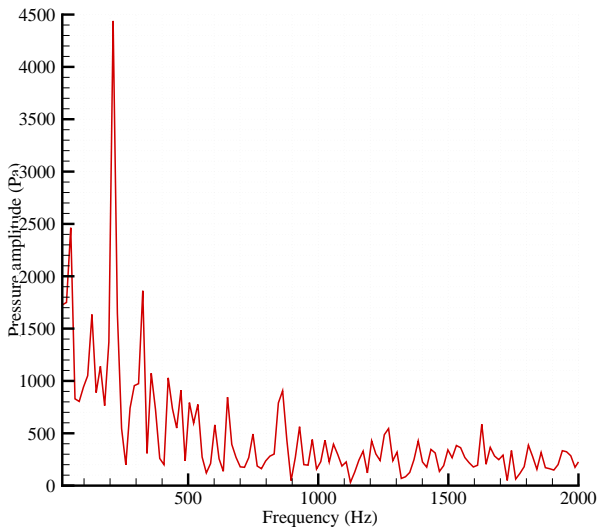

(d) Rear cavity wall at $\mathrm{y} / \mathrm{D}=0.50$.

Figure 11. Empty cavity pressure spectra at floor and rear wall centreline, $L / D=5.42$ and $W / D=2$. 


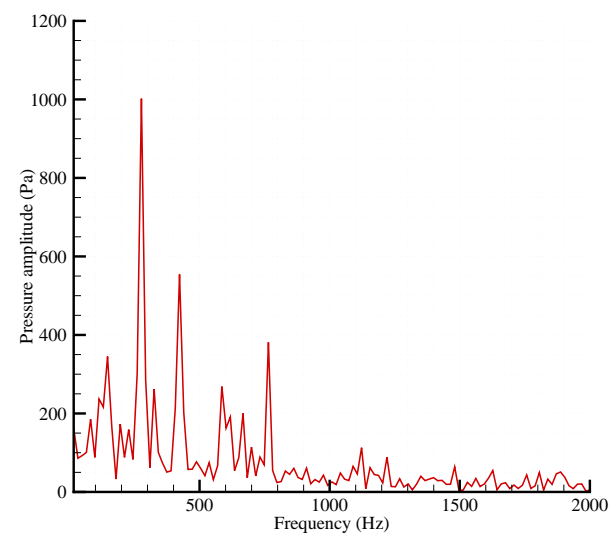

(a) Floor at $\mathrm{x} / \mathrm{L}=0.10$.

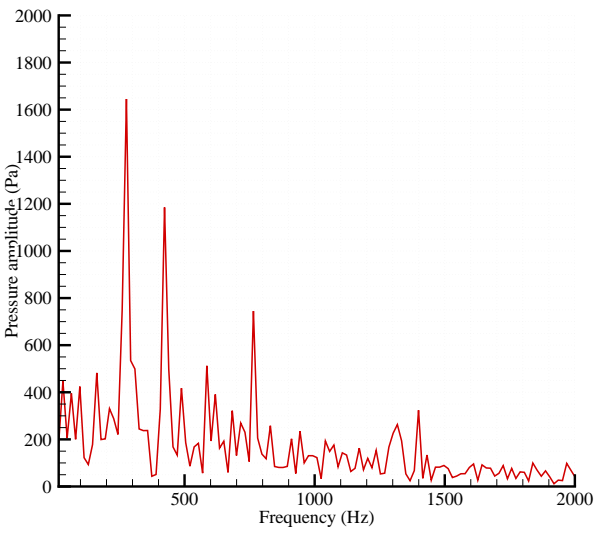

(c) Floor at $\mathrm{x} / \mathrm{L}=0.667$.

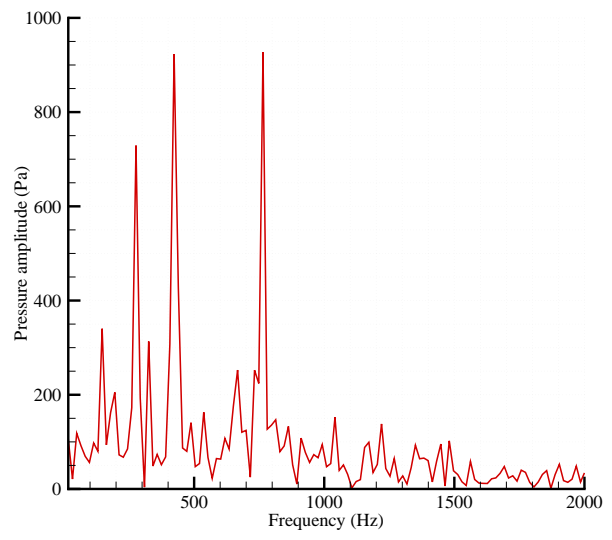

(b) Floor at $\mathrm{x} / \mathrm{L}=0.30$.

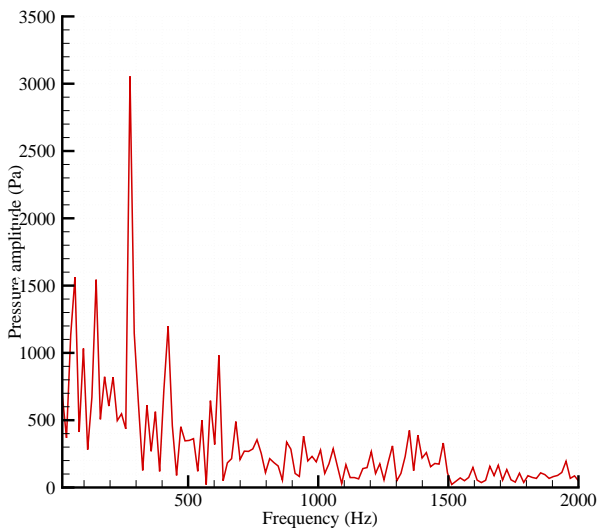

(d) Floor at $\mathrm{x} / \mathrm{L}=0.90$.

Figure 12. Cavity with store pressure spectra at floor, $L / D=5.42$ and $W / D=2$.

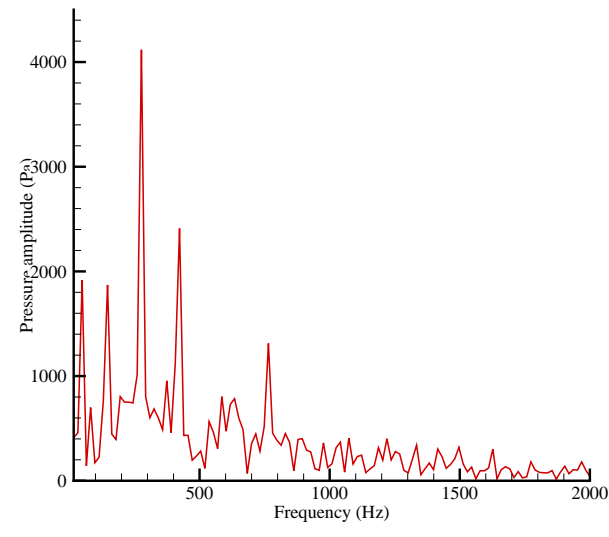

(a) Rear wall at $\mathrm{y} / \mathrm{D}=0.25$.

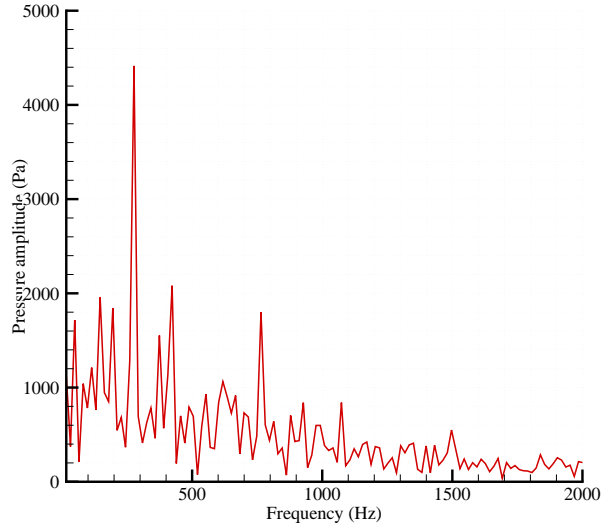

(b) Floor at $\mathrm{y} / \mathrm{D}=0.50$.

Figure 13. Cavity with store pressure spectra at rear wall centreline, $L / D=5$ and $W / D=2$. 


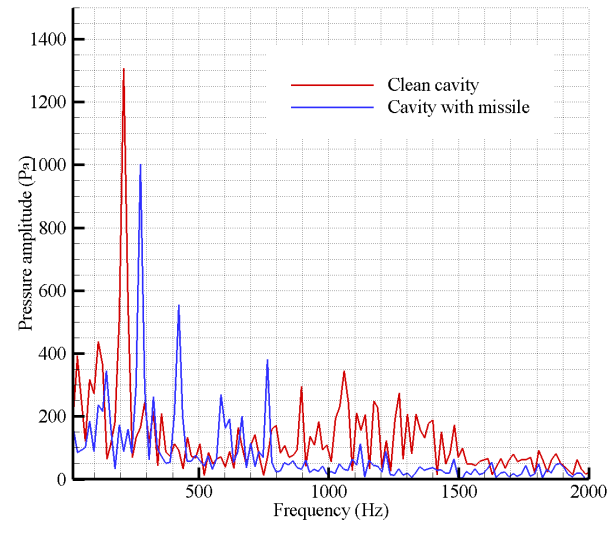

(a) Floor at $\mathrm{x} / \mathrm{L}=0.10$.

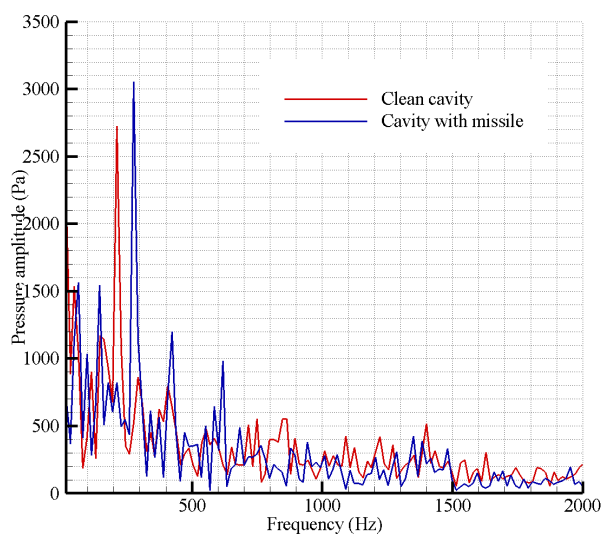

(b) Floor at $\mathrm{x} / \mathrm{L}=0.90$.

Figure 14. Floor centreline pressure spectra comparison for clean cavity and cavity with store, $L / D=5$ and $W / D=2$.

case.

The mean flowfield visualisation in the time-mean flow study clearly showed that the flowfield was divided in two halves with little evidence of flow across the store and supporting strut. This blockage effect of store and strut in the spanwise flow might have reduced the possibility of spanwise and streamwise flow fluctuations undergoing non-linear coupling to result in amplitude modulations. This may be the reason for the co-existence of multiple modes without the coupling among them.

Table 1. Comparison of mode frequencies $(\mathrm{Hz})$ for cavity with $L / D$ ratio of 5.42

\begin{tabular}{cccc}
\hline \hline mode & Clean cavity & Cavity with store & Rossiter Formula \\
\hline 1 & 114 & 145 & 111.4 \\
\hline 2 & 212 & 276.6 & 279 \\
\hline 3 & - & 424 & 445 \\
\hline 4 & - & 618 & 615 \\
\hline 5 & - & 765 & 782 \\
\hline \hline
\end{tabular}

\section{Conclusion}

The time-mean cavity flowfield was studied computationally. Significant spanwise flow was found to exist for the 3D clean cavity. Mean pressure coefficients were also compared for a clean 
cavity and a cavity with store (both with $L / D$ of 5.42) against experimental data and the result showed reasonably good agreement between the two. The clean cavity and the cavity with a store case have negligible difference in their $C_{p}$ distribution on the front half of the cavity whereas the difference was more pronounced at the rear half. The presence of a store is seen to limit the spanwise flow in the cavity and this is thought to have caused this small difference. A small difference is found to exist between the experimental and the computational results. This is thought to be mainly due to the influence of differences in the nature of the boundary layers at the cavity leading edge (in the computational setup and experiment) and its effect on the instability characteristics of shear layers that span the cavities.

Unsteady flow results were also analysed for all three cases. For the first case, the computed frequency of the dominant mode is in good agreement with the experiment. For the second case, first mode frequency from clean cavity case is found to agree well with the modified Rossiter formula. The dominant mode, however, is not the first mode and the dominant peak occurs at 212 Hz. This frequency is significantly lower compared to the second mode of $279 \mathrm{~Hz}$ predicted by the modified Rossiter formula. Higher order modes are absent in the spectra. In the case of high speed cavity flows, existence of more than single dominant modes is likely and these multi-modes were found to couple non-linearly resulting in low frequency modulation of the Rossiter modes. This is thought to be the main reason for the presence of the dominant mode at $212 \mathrm{~Hz}$. Further studies are ongoing to understand this better.

Study of unsteady pressure spectra for the last case found the presence of many peaks and the corresponding mode frequencies are found to agree well with the Rossiter modes. Up to five Rossiter mode were found to be present for this case, in contrast to clean cavity case. The mean flowfield visualisation in the time-mean flow study clearly showed that the flowfield was divided in two halves with little evidence of flow across the missile and supporting strut. This blockage effect of missile and strut on the spanwise flow might have reduced the interaction of spanwise and streamwise flow fluctuations undergoing non-linear coupling to result in amplitude modulations. This may be the reason for the co-existence of multiple modes without the coupling among them. Further study is ongoing for the cavity-with-store case. 


\section{References}

${ }^{1}$ Dix, R. E. and Bauer, R. C., "Experimental and predicted acoustic amplitudes in a rectangular cavity," No. AIAA-2002-0472, 2000.

${ }^{2}$ Srinivisan, S. and Baysal, O., "Navier-Stokes calculations of transonic flow past cavities," Journal of Fluid Engineering, Vol. 113, September 1991, pp. 369-376.

${ }^{3}$ Tam, C. K. W. and Block, P. J. W., "On the tones and pressure oscillations induced by flow over rectangular cavities," Journal of Fluid Mechanics, Vol. 89, 1978, pp. 373-399.

${ }^{4}$ Stallings, R. and Wilcox, F., "Experimental cavity pressure distributions at supersonic speeds," Tech. Rep. NASA-TP-2683, NASA, June 1987.

${ }^{5}$ Xiao, X., Edwards, J. R., and Hassan, H. A., "Blending Functions in Hybrid Large-Eddy/Reynolds-Averaged NavierÜStokes Simulations,” AIAA Journal, Vol. 42, No. 12, 2004, pp. 2508-2515.

${ }^{6}$ Spalart, P. R. and Allmaras, S. R., "Comments on the feasibility of LES for wings and on a hybrid RANS/LES approach," Proceedings of first AFOSR international conference on DNS/LES, Ruston, Louisiana, USA, August 1997.

${ }^{7}$ Nichols, R. H., "Comparison of Hybrid Turbulence Models for a Circular Cylinder and a Cavity," AIAA Journal, Vol. 46, No. 6, 2006, pp. 1207-1219.

${ }^{8}$ Sinha, N., Dash, S., Chidambaram, N., and Findlay, D., "A Perspective on the Simulation of Cavity Aeroacoustics," No. AIAA-1998-0286, Jan. 1998.

${ }^{9}$ Spalart, P. R., Deck, S., Shur, M. L., Squires, K. D., Strelets, M. K., and Travin, A., "A new version of detachededdy simulation, resistant to ambiguous grid densities," Theoritical and Computational Fluid Dynamics, Vol. 20, 2006, pp. 181-195.

${ }^{10}$ Charwat, A. F., Roos, J. N., Dewey, F. C., and Hitz, J. A., "An Investigation of Separated Flows Part 1 : The Pressure Field," Journal of Aerospace Sciences, Vol. 28, No. 6, 1961, pp. 457-470.

${ }^{11}$ Charwat, A. F., Roos, J. N., Dewey, F. C., and Hitz, J. A., "An Investigation of Separated Flows Part 2 : Flow in the Cavity and Heat Transfer," Journal of Aerospace Sciences, Vol. 28, No. 7, 1961, pp. 513-527.

${ }^{12}$ Plentovich, E., "Three-dimensional cavity flow fields at subsonic and transonic speeds," Tech. Rep. NASATM-4209, NASA Langley Research Center, 1992.

${ }^{13}$ Tracy, M. B. and Plentovich, E., "Measurements of fluctuating pressure in a rectangular cavity in transonic flow at high Reynolds number," Tech. Rep. NASA-TM-4363, NASA Langley Research Center, 1992.

${ }^{14}$ Ritchie, S. A., Non-Intrusive Measurements and Computations of Transonic Cavity Flows with Applications to Aircraft Stores Release, Ph.D. thesis, Cranfield University, Sept 2005.

${ }^{15}$ Jr., R. L. S., Plentovich, E. B., Tracey, M. B., and Hemsch, M. J., "Measurements of store forces and moments and cavity pressures for a generic store in and near a box cavity at subsonic and transonic speeds," Tech. Rep. NASATM-4611, NASA, May 2002.

${ }^{16}$ Tracy, M. B. and Plentovich, E., "Characterization of cavity pressure flow fields data obtained in Langley $0.3 \mathrm{~m}$ Transonic Cryogenic Tunnel,” Tech. Rep. NASA-TM-4436, NASA Langley Research Center, 1993.

${ }^{17}$ Tracy, M. B. and Plentovich, E. B., "Cavity unsteady-pressure measurements at subsonic and transonic speeds," Tech. Rep. NASA-TP-3669, NASA Langley Research Center, 1997.

${ }^{18}$ Kim, S., Dai, Y., Koutsavdis, E. K., Sovani, S., Kadam, N. A., and Ravuri, K. M. R., "A Versatile Implementation of Acoustic Analogy Based Noise Prediction Method in a General-Purpose CFD Code," No. AIAA-2003-3202, $9^{\text {th }}$ AIAA/CEAS Aeroacoustics Conference and Exhibit, Hilton Head,South Carolina, USA, May 2003.

17 of 18

Computational study of cavity flowfield at transonic speeds, Khanal et al. 
${ }^{19}$ Mathey, F., Morin, O., Caruelle, B., and Debatin, K., "Simulation of aeroacoustic sources in aircraft climate control systems," No. AIAA-2006-2493, $12^{\text {th }}$ AIAA/CEAS Aeroacoustics Conference and Exhibit, Cambridge, Massachusetts, USA, May 2006.

${ }^{20}$ Joeng, J. and Hussain, F., "On the identification of a vortex," Journal of Fluid Mechanics, Vol. 285, 1995, pp. 69-94.

18 of 18

Computational study of cavity flowfield at transonic speeds, Khanal et al. 